\title{
A simple statistical approach that represents the frequency distribution of plasmids in clinical isolates of the enterobacteria
}

\author{
D. J.PLATT
}

\section{University Department of Bacteriology, Royal Infirmary, Castle Street, Glasgow G4 OSF}

\begin{abstract}
Summary. The frequency distribution of plasmids in a representative collection of Escherichia coli and other enterobacteria was compared with the frequencies predicted by the Poisson distribution. The distribution of $E$. coli plasmids did not differ significantly $(p>0.2)$ whereas the difference between observed and predicted distributions of plasmids in combined populations of other enterobacteria was significant $(\mathrm{p}<0 \cdot 001)$. Previous studies had suggested that plasmid-free strains contributed disproportionately to the overall frequency distribution. Therefore, plasmid-free strains were excluded and the frequency distribution of plasmids in plasmid-containing strains compared with frequencies predicted by a modified Poisson distribution conditional upon $n \neq 0$. The results obtained showed that the frequency distribution of plasmids in $E$. coli and in other enterobacteria did not differ significantly from the predicted distribution ( $p>0.2$ and $p>0.6$ respectively). The frequency distribution of plasmids in previously published studies was compared with predicted frequencies by means of (a) the Poisson distribution and (b) the modified Poisson distribution conditional upon $n \neq 0$. The results indicate that the modified Poisson distribution described provides an adequate description of plasmid frequency distributions and represents the observed frequencies better than the distributions predicted directly from the Poisson formula.
\end{abstract}

\section{Introduction}

The overall distribution of plasmids in natural populations of the enterobacteria reflects the interaction of many factors. These include plasmid mobility, host range, selection pressure, recipient ability and the prevailing physiological conditions (Anderson, 1975; Richmond and Petrocheilou, 1978; Sanderson et al., 1981; Reanney et al., 1983). Some of these have been investigated individually in vivo and in vitro, and models have been devised that have considered the relationships between different factors (Cullum et al., 1978; Levin et al., 1979; Freter et al., 1983). An alternative and complementary approach would consider the end product of these diverse interactions, namely the frequency distributions of plasmids in different bacterial populations.

In a previous report (Platt et al., 1986b) we described the frequency distribution of plasmids among a representative collection of enterobacteria and compared Escherichia coli with the other

Received 1 July 1986; accepted 4 Aug. 1986. enterobacteria. Significantly more plasmids were found in $E$. coli. However, when the distribution of plasmids was considered as a function of strains that harboured plasmids (i.e., plasmid-free organisms were excluded from both groups) there was no significant difference between the distributions. We suggested that other enterobacteria contain a higher proportion of strains less able to accumulate plasmids than $E$. coli. The general appearance of these and other distributions (Platt et al., 1984) suggested that they might follow a Poisson distribution. However, the hypothesis relating to differences in recipient ability implies that a given collection of bacterial strains is not homogeneous and, as a result, is not amenable to direct comparison with Poisson predictions. This report compares the frequency distribution of plasmids in several collections of $E$. coli and other enterobacteria (Platt et al., 1984, 1986a and $b$ ) with values predicted from a Poisson distribution. A modification of the Poisson formula necessary to allow the calculation of predicted frequencies after the exclusion of plasmid-free strains is described. 


\section{Materials and methods}

The organisms, plasmids and the frequency distribution of plasmids have been described previously and are summarised in table I.

\section{Statistical analysis}

A random variable $X$ (the number of plasmids per bacterial strain) is said to have a Poisson distribution if the probability that a given strain will harbour " $n$ " plasmids is given by

$$
\mathrm{P}(\mathrm{X}=\mathrm{n})=\frac{\mathrm{e}^{-\lambda} \lambda^{\mathrm{n}}}{\mathrm{n} !} \quad(\mathrm{n}=0,1,2, \ldots, \mathrm{n} ; \lambda>0)
$$

where the parameter $\lambda$ is the mean value of $X$.

The Poisson distribution can be modified, conditional upon $\mathrm{n} \neq 0$ to give

$$
\begin{aligned}
& P(X=n \text { given } X \neq 0)= \\
& \qquad \frac{e^{-\lambda} \lambda^{n}}{n !\left(1-e^{-\lambda}\right)}(n=1,2, \ldots, n ; \lambda>0)
\end{aligned}
$$

The mean value of $X$, given $X \neq 0$ can be shown to be

$$
\frac{\lambda}{1-\mathrm{e}^{-\lambda}}=\lambda^{\prime}
$$

Since equation (3) is not readily solved for $\lambda, \lambda^{\prime}$ was calculated for values of $\lambda$ between $0 \cdot 1$ and 4.0 and are shown graphically in fig. 1 . From this graph, values of $\lambda$ were determined from experimental values of $\lambda^{\prime}$. For the bacterial collections and sub-populations (table I) the frequency of isolates that harboured $0-n$ plasmids was predicted from equation 1 . After the exclusion of plasmidfree isolates $\lambda^{\prime}$ was calculated and $\lambda$ determined by reference to fig. 1. The frequency of isolates that harboured plasmids $(1-n)$ was predicted from equation 2.

The "Goodness-of-fit" of the Poisson distribution to the experimental data was tested by referring the value of $\chi^{2}=\Sigma(\mathrm{Obs}-\operatorname{Exp})^{2} /$ Exp to a $\chi^{2}$ distribution on $(\mathrm{k}-$ $\mathrm{p}-1)$ degrees of freedom where

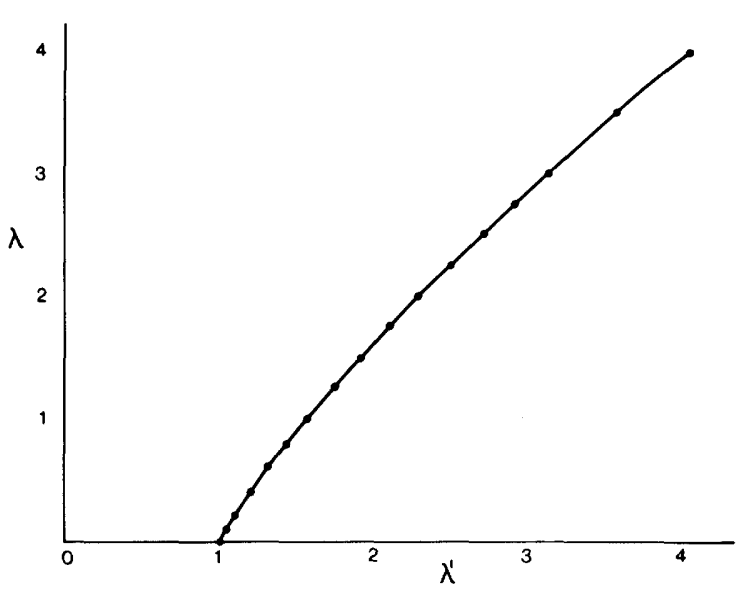

Fig. 1. Graph of mean value $(\lambda)$ of a Poisson-distributed random variable $(\mathrm{X})$ against the mean value $\left(\lambda^{\prime}\right)$ obtained conditional upon $X \neq 0$.

$k=$ number of categories after grouping to ensure that no predicted value was $<2$

$p=$ number of parameters estimated from the data $(=1$ since $\lambda$ was estimated)

In all cases the last category was interpreted as $; \geqslant X$ and predicted values adjusted accordingly so that $\Sigma$ observed $=\sum$ Expected.

\section{Results and discussion}

The comparison of plasmid frequency distributions from different collections and sub-populations of enterobacteria with frequencies predicted from a Poisson distribution is shown in table II. Most (8 out of 11) showed significant differences. However, no significant difference was found for unselected E. coli strains from the gut, Salmonella typhimurium, and the drug-sensitive sub-population of $E$. coli

Table I. Collections of enterobacteria studied

Characteristics of collection/sub-population
Designation

$\begin{array}{ll}\text { A } & \text { Platt } \text { et al. }(1986 b) \\ \text { B } & \\ \text { C } & \text { Platt } \text { et al. }(1986 a) \\ \text { 1, D2 } & \\ \text { 1, E2 } & \\ \text { G , F2 } & \text { Platt et al. }(1984) \\ \text { 1, H2 } & \\ \text { I } & \end{array}$

Reference
Unselected $E$. coli isolated from the gut

Unselected other enterobacteria isolated from the gut

$S$. typhimurium selected only by the exclusion of multiple isolates from the same source or outbreak

$E$. coli isolated from wounds and the gut of patients with vascular disease

Sensitive sub-population of D

Resistant sub-population of D

Sub-population of $\mathrm{F}$ that showed transferable resistance

Urinary E. coli strains collected on the basis of trimethoprim resistance

Sub-population of $\mathrm{H}$ that showed transferable resistance
1 refers to the distribution of all plasmids detected; 2 refers to the distribution of plasmids of mol. wt $>20 \times 10^{6}$. 
FREQUENCY DISTRIBUTION OF PLASMIDS

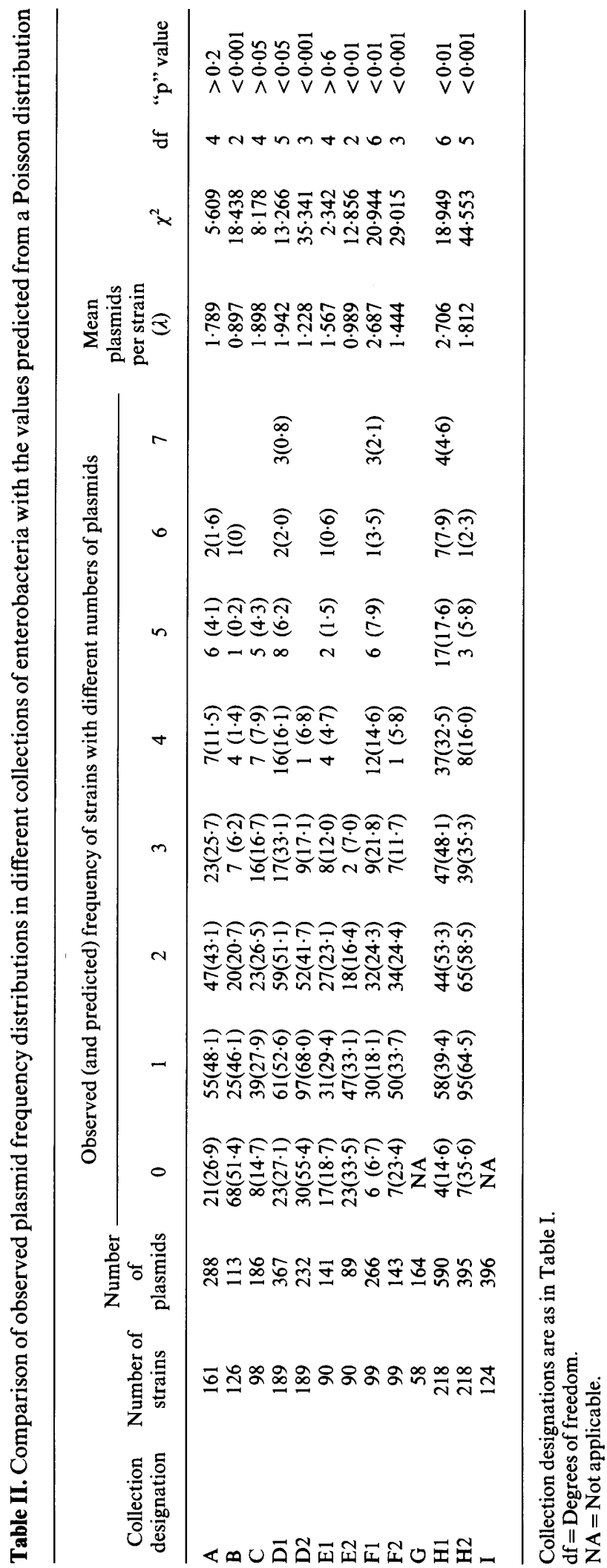




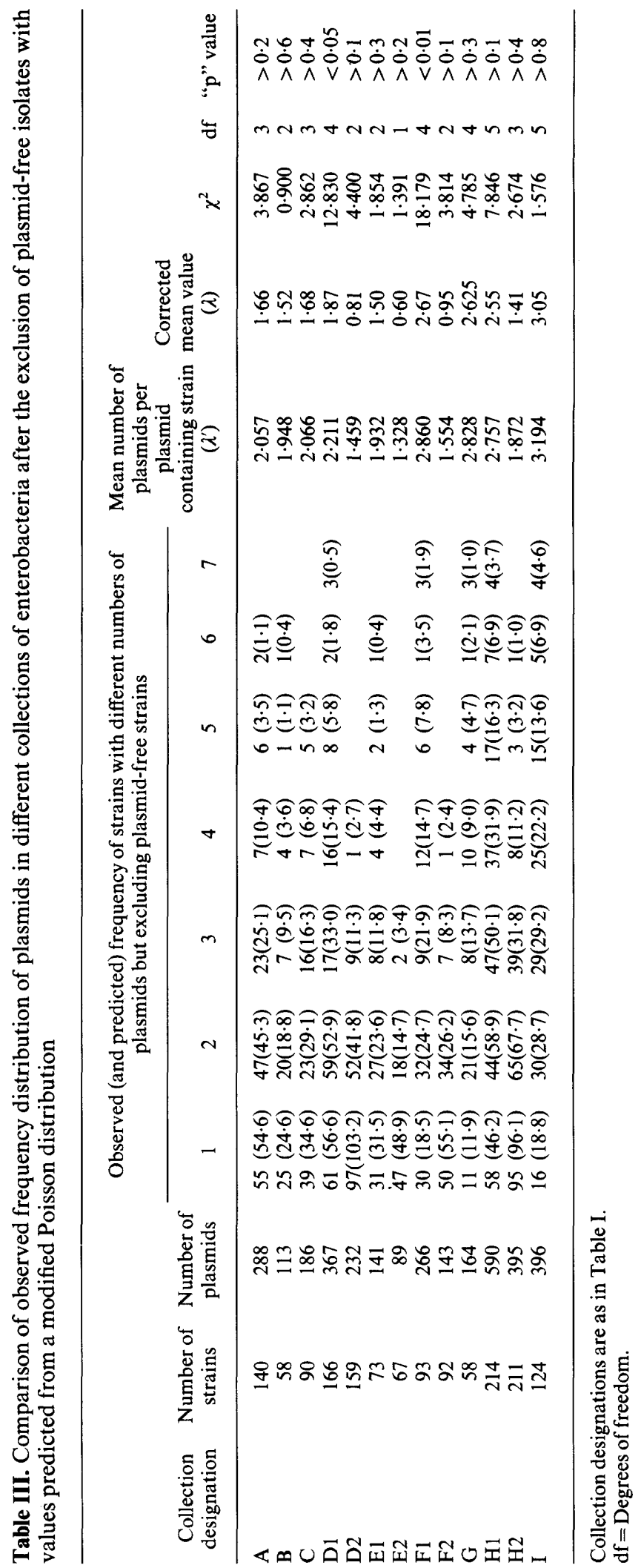


isolated from patients with vascular disease. These results indicate that plasmid frequency distributions are not well represented by the Poisson distribution. When similar comparisons were made after the exclusion of plasmid-free strains (table III) only two of the 13 observed frequency distributions differed from the distribution of frequencies predicted by the modified Poisson formula which, therefore, adequately represents the distribution of plasmids in strains that contain them. Considering individual results in relation to this, the observation that $E$. coli and $S$. typhimurium (A, C or E1; table I) did not differ significantly from the predicted values obtained from the unmodified Poisson distribution suggests that these collections contained few poor recipients. The observed plasmid frequencies among most of the collections and sub-populations did not differ significantly from the values predicted by the modified Poisson distribution. However, one collection and sub-population of $E$. coli from patients with vascular disease did differ (D1 and F1; table I). The five sub-populations of this collection that did not differ from the results predicted by the modified Poisson distribution, either specifically (D2, E1, E2 and F2) or implicitly (G) excluded resistant strains that did not harbour large (mol. wt $\left.>20 \times 10^{6}\right)$ plasmids. Therefore, it seems likely that small plasmids may be responsible for the observed deviations. Moreover, studies with restriction fingerprints to characterise the strains further (J. S. Chesham, unpublished data) have shown that the original collection inadvertantly included a small number of duplicate isolates, which may explain the observed distortion of the distributions.

Two further observations are noteworthy. First, the two sub-populations ( $G$ and $I$ ) that implicitly included no plasmid-free isolates (on the basis that all transferred drug resistance by conjugation) did not differ from the predictions of the modified Poisson. Second, the finding that the distribution of plasmids in a collection selected on the basis of trimethoprim resistance were well represented by the modified Poisson predictions was unexpected. However, this collection contained a large proportion of multi-resistant strains (Kraft et al., 1983) and may not be typical of drug-resistant subpopulations in general.

Thus, although many different factors influence the acquisition and accumulation of plasmids among enterobacterial populations, it appears that, with the exception of recipient ability, the overall process is a random one. The analysis of plasmid distributions as described may have several clinical applications. First, in any local situation where plasmids are routinely monitored, the occurrence of cross-infection episodes will often cause detectable distortion of the distribution. This will depend on the individual distribution and the number of plasmids harboured by the cross-infecting organism. Second, where strains that harbour one or more plasmids that confer the ability to colonise or invade a specific anatomical site, the distribution of plasmids among a collection of strains isolated from that site may be expected to differ from the frequencies predicted by the modified Poisson distributions.

On the basis of these findings the following model is proposed for the distribution of plasmids among the enterobacteria. A representative collection of enterobacterial strains comprises two overlapping sub-populations (fig. 2). The first sub-population is plasmid-free and the second possesses plasmids distributed according to the Poisson distribution. The region of overlap represents that proportion of strains that do not harbour plasmids but are capable of doing so whereas the component of the first subpopulation that lies outwith the overlapping region represents strains less able to acquire or maintain them.

In a previous study (Platt and Sommerville, 1981) we found that of 46 strains of Enterobacter spp. tested for recipient ability, only a minority were efficient and most of these already harboured plasmids whereas poor recipients were generally

\section{Plasmid free sub-POPUlation}
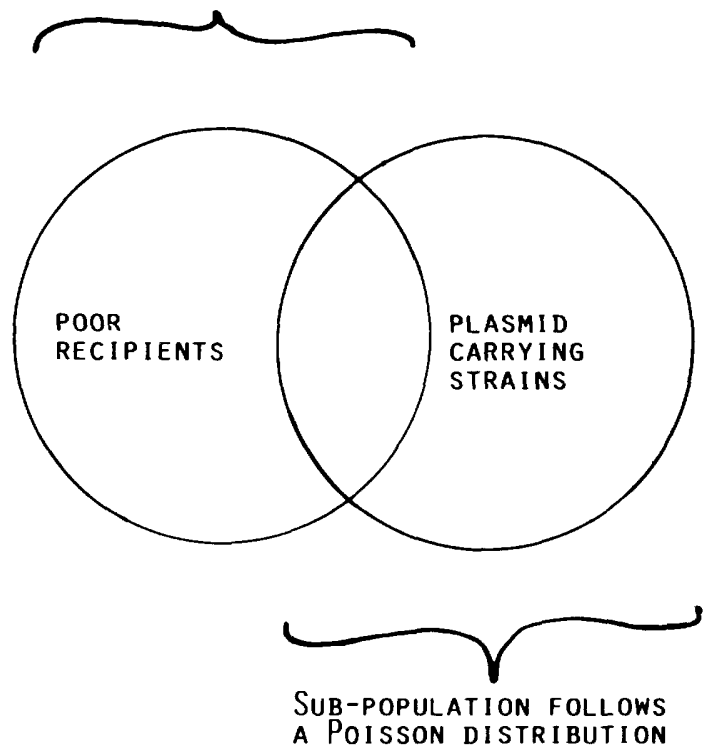

Fig. 2. Proposed model for the distribution of plasmids in a representative collection of enterobacteria. 
plasmid-free. These observations lend indirect support to the model.

I thank W. Harper Gilmore of the Department of Medical Statistics for critically reading the manuscript.

\section{REFERENCES}

Anderson J D 1975 Factors that may prevent transfer of antibiotic resistance between Gram-negative bacteria in the gut. Journal of Medical Microbiology 8:83-88.

Cullum J, Collins J F, Broda P 1978 Factors affecting the kinetics of progeny formation with F'lac in Escherichia coli K12. Plasmid 1:536-544.

Freter R, Freter R R, Brickner H 1983 Experimental and mathematical models of Escherichia coli plasmid transfer in vitro and in vivo. Infection and Immunity 39:60-84.

Kraft C A, Platt D J, Timbury M C 1983 Distribution and transferability of plasmids in trimethoprim-resistant urinary Escherichia coli. Journal of Medical Microbiology 16: 433-441.

Levin B R, Stewart F M, Rice V A 1979 The kinetics of conjugative plasmid transmission: fit of a simple mass action model. Plasmid 2:247-260.
Platt D J, Brown D J, Munro D 1986a Distribution of plasmids in a representative collection of Scottish salmonellae. Journal of Hygiene 97:199-204.

Platt D J, Chesham J S, Kristinsson K 1986b R-plasmid transfer in vivo: a prospective study. Journal of Medical Microbiology 21:325-330.

Platt D J, Sommerville J S 1981 A simple method for the detection of resistance plasmids in Serratia species by transfer to members of the genus Enterobacter. Journal of Antimicrobial Chemotherapy 8:145-152.

Platt D J, Sommerville J S, Kraft C A, Timbury M C 1984. Antimicrobial resistance and the ecology of Escherichia coli plasmids. Journal of Hygiene 93 : 181-188.

Reanney DC, Gowland P C, Slater J H 1983 Genetic interactions among microbial communities. In: Slater $\mathbf{J} \mathrm{H}$ et al. (eds) Microbes in their natural environments. (Society for General Microbiology Symposium 34). Cambridge University Press, Cambridge, pp 378-421.

Richmond M H, Petrocheilou, V 1978 R-factor transfer in vivo in humans. In: Schlessinger D (ed) Microbiology-1978American Society for Microbiology, Washington, DC, pp 273-276.

Sanderson K E, Janzer J, Head J 1981 Influence of lipopolysaccharide and protein in the cell envelope on recipient capacity in conjugation of Salmonella typhimurium. Journal of Bacteriology 148 : 283-293. 